\title{
Trust and Closeness: A Mixed Method for Understanding the Relationship of Social Network Users
}

\author{
Lei Yang \\ Shandong University of Science and \\ Technology \\ yanglei@sdust.edu.cn
}

\author{
Xue Wang \\ Shandong University of Science and \\ Technology \\ 17854259823@163.com
}

\author{
Margaret Meiling Luo \\ National Chung Cheng University \\ luo@mis.ccu.edu.tw
}

\begin{abstract}
Mutual trust among social network users encourages positive communications; it is critical to study trust in the context of online social networks. We built a trust model and crawled data according to a closeness index. Data was collected from Qzone, an $S N$ service (also known as QQ) in China. We identified 150 QQ users and 3 friends from each of the users; data of these users were collected by Python program. The relationship between trust and closeness was constructed using an ordinary least squares regression model, and the factors that influence trust between social network users were constructed using an endogenous switching regression model. We found that user trust and closeness are positively correlated. A user's trust is positively related to three closeness indicators: comments, @s to QQ friends (a reminder nudge for attention), and messages. Increasing closeness in social networks has a positive effect on trust formation.
\end{abstract}

Keywords: Social network, Trust, Closeness, Endogenous Switching Regression

\section{Introduction}

Social networking (SN) refers to the practice of expanding one's social contacts by using social media to connect with individuals. The development of the Internet has enabled social media to provide a variety of functions. Users are able to share thoughts, post photos, and leave comments using social media. In China, QQ and WeChat are the most popular social networking services (SNS). In December 2018, the number of SNS users in China had reached 792 million, 95.6\% of the total number of Chinese Internet users [1].

Positive SNS communication is built upon a trust relationship between users. However, trust over SNS media is impeded by a number of factors: 1) the complexity and instability of social networks, 2) existence of false and malicious users, 3) risk of online fraud, and 4) dissemination of false information. Thus, building a solid trust relationship over the Internet is challenging and an increasingly prominent problem that calls for the attention of researchers.
It is an important research agenda to understand the linkage between observed tie-strength measures and trust. Although researchers has found that trust in known entities can be transferred to business transactions facilitated through a social network with unknown parties (e.g. Sharma, Menard and Mutchler, 2019), little do we know to what extent trust is understood in terms of technology enabled size, scale, and reach of online social networks in emerging market such as China. The value of understanding trust in this case will benefit the sharing economy business models where users deal with friends/other users in a virtual space. We aim to provide valuable information to design of the computational trust systems that have capacities to enable peer-to-peer sharing platforms of the new economy.

Logically, measures of friendship and social tie on social media may function differently online versus how it is in the physical world. For instance, the ease of "friending" an online friend lead an issue of understanding latent heterogeneity about level of trust to users' online friends how trusting users are of their friends. We are also interested to know whether level of trust varies with the number of social media friends they have. To know how trust work, we shall focus on the linkage between both the dynamic and behavioral strength of tie measures in social media. In addition, levels of trust between friends is crucial because economic and social capital exists in in populationscale online networks. The values of the capital is enormous and can be expected.

The goal of this research is to understand how trust perceived by social media users. Especially, we are interested in factors that influence trust between adjacent users in social networks. We intend to identify the interaction between trust and closeness of social media users. Through collecting online data, we aim to investigate how a user's trust can be built via improving closeness with his or her social media friends. We also intend to understand whether users who have more interactions and those who have fewer interactions are similar or different and how they improve trust between each other. 
Here, we investigate and propose a threedimensional model that focuses on 1) characteristic similarity trust, 2) interaction trust, and 3) platform evaluation trust. The research procedure for this paper is shown in Figure 1.

\section{Literature and theoretical background \\ 2.1. Studies on trust}

Trust has various definitions. For instance, from a philosophical perspective, Baier [2] proposed that trust is an emotional attitude. He argued that trust can be morally valuable in four areas of ethical life: moral development, moral identity, moral perception and judgement, and living a good life. He further noted that trust is often related to social values or interests and is often inextricably linked to interpersonal trust. Antoci et al. [3] found that participation in political or social activities can promote interpersonal trust between individuals. In management, trust is defined as an informal characteristic of governance and is also seen as a key factor in the development of an organization [4]. Strauss [5] constructed conceptual models informed by psychology, economics, sociology, and public relations and studied the prominence of trust in investor relations. Yadav, Chakraverty, and Sibal [6] referring to applicability and extensibility, refined the concept of trust into similarity-based trust and relationship-based trust. In business, trust is defined as a person's confidence in the reciprocal party's capability and willingness to adhere to relationship norms and keep promises [7].

Developing trust in real life is different from how it is done in a social networking environment. In real life, people build trust with others through long-term relationships, and trust is often based on friendship and kinship. In social networks, frequent interactions between users and their friends, sharing, or brief connections made for business may create a sense of trust between users.

Social networking scholars have also studied trust. In social media research, investigators have combined social network analysis and experimental economics [8] to study how social networks affect trust and trustworthiness [9]. Other researchers have analyzed mechanisms for estimating and disseminating trust and reputation in distributed network settings. They summarized measures based on network communication mechanisms and combined these measures with decision-making strategies, in an effort to use trust-based social networks to promote decisionmaking and recommendation processes [10]. Sabatini and Sarracino [11] used survey data to explore the effect of participation in social networks on social capital and trust. They discovered that an increase in online network participation negatively affected three types of trust: social trust, specific trust, and institutional trust. They also found that the social characteristics of senders and receivers can predict trust and distrust. Akilal, Slimani, and Omar [12] proposed an algorithm that could predict trust and distrust based on adjacent users of the trustor and the trustee. Finally, Golzardi, Sheikhahmadi, and Abdollahpouri [13] used three indicators-user trust, similarity, and personal reputation-to predict the strength of the trust relationship between users.

Trust and forgiveness both play crucial roles in social network communication. Laifa, Akrouf, and Mammeri [14] used a two-stage approach to study how potential forgiveness influences the maintenance of connectedness. They used structural equation modeling (SEM) to test their model then used the results as input for artificial neural network and fuzzy logic models to provide more accurate predictions. They also created an agent-based simulation to show the possible implementation of the models. Frey, Buskens, and Corten [15] organized 342 participants to conduct a repeated trust game with endogenous and exogenous embeddedness. They found that either form of embeddedness promotes trustfulness and trustworthiness and trustors and trustees invest in embeddedness in trust problems but endogenous embeddedness has stronger effects than exogenous embeddedness. Xu et al. [16] constructed a privacy protection mechanism based on social network trust. They performed a simulation, and the results demonstrated that a trust-based photo-sharing mechanism helps reduce loss of privacy, which can enhance user trust in social networks. Their proposed threshold-tuning method can balance privacy loss and information shared with others.

\subsection{Social network closeness}

Closeness can be defined as "affective or emotional interdependence that contains such relational properties as liking, trusting and respecting one another" [17]. In research on social network closeness, Asim et al. [18] proposed the "SNTrust" model to discover the trust of nodes in a network and to study trust, influence, and the relationship of these in a social network. To overcome the problems of trust prediction in online social rating networks, Ali-Eldin [19] introduced a new global trust computation model that uses trusted parties' recommendations to weigh user ratings. Ntwiga, Weke, and Kirumbu [20] aimed to model user trust of agents. They used peer-to-peer reputation ratings in a network and concluded that 
performing singular value decomposition when estimating trust from reputation ratings is an ideal approach to error elimination. Bapna et al. [21] used a customized Facebook program to explore how social strength is associated with trust metrics. They argued that because not all online social ties are created equal, traditional measures of dyadic trust such as embeddedness may not always be effective predictors of digital trust. To support workflow, Park et al. [22] proposed an algorithm that analyzes social network closeness centrality to represent collaborative relationships among social network users participating in a particular workflow model. Khopkar et al. [23] proposed a fast incremental update algorithm that calculates the shortest path, closeness centrality, and intermediary centrality of all user nodes. They suggested that the size of the personal network and the scale of the extended network are positively related to the diversity of people's social networks. Shen and Gong [24] investigated WeChat and reported that it can provide users with opportunities to accumulate a wide range of relationships, including strong and weak relationships. Lin et al. [25] proposed a filter and operational framework to handle dynamic trends in large-scale social networks and then conducted an experiment in real networks. They concluded that centrality can be used to measure the activity of social network nodes and improve communications. Zhang and Luo [26] elaborated hierarchical centrality, intermediary centrality, and closeness centrality, presenting these centralities from principle to algorithm. Finally, Moore, Carrasco, and Tudela [27] studied the effects of individual interaction attributes on the duration of, distance of, and number of people involved in daily activities in society through SEM methods. They found that social closeness, gender, age, and network density help to prolong the duration of online social activities.

\subsection{Research gap in trust studies}

Researchers have been modeling trust in social networks from a variety of perspectives. Using various models and methods of calculation, their purpose has been to predict or calculate trust in social networks. Research on relationship closeness has typically explored real situations through modeling or empirical analysis. We found numerous studies of social networks that used modeling, but few of these explored the factors that affect trust in social networks and the relationship between such trust and these factors. Thus, we built a trust calculation model and then conducted an empirical study. Our goal was to improve our understanding of the role of closeness in the formation of trust in social networks and the relationship between trust and closeness in social networks.

\section{Construction of trust calculation model and variables setting \\ 3.1 Data collection}

We recruited 150 students, a prominent demographic among social network users, from a university in eastern China. Participants are university students who major in science and engineering, humanities and social sciences. All the participants are psychically located in Qingdao, Shandon. There are a total of 25 post-secondary institute. The city Qingdao is the economic center of Shandon province. Major industries include light industry, tourism service, and Aquaculture.

The participants were recruited through online advertising. A random sampling scheme was used (see Table 1 for demographics and QQ use experience). We collected their trust score data from Qzone (Tencent Technology Co., Ltd.), an SN service. QQ is a major social network service in China, listed together with WeChat as the most popular SNS • In 2019, the active user in QQ is 647 million [41]. As of March 2020, social network Website, Qzone, accounts for $47.6 \%$ SNS users [42].

The group comprised 108 seniors, 24 juniors, and 18 first-year graduate students who were heavy users of QQ (defined as having a QQ level of between 32 and 80 and having been active QQ users for between 1152 and 6720 days). QQ level reflects experience with Qzone's SN site. After obtaining consent of these 150 participants, three QQ friends of these participants were randomly selected as our observations. We used a Python program to crawl data and obtained 422 sets of valid data, including comments, messages, @s to QQ friends, QQ level, and gender. The data suggested that an adjacent user trust calculation model could enable us to estimate quantitative trust. We then conducted an empirical analysis using the data.

\subsection{Trust calculation model 3.2.1 Model construction}

Zhan and Fang [28] constructed a trust calculation model, accounting for three domains: attribute similarity, information reliability, and social evaluation. To measure quantitative trust in social networks, we intended to improve upon their model. We proposed that an individual tends to trust an individual who is similar to them, that frequent interactions increase mutual trust, and that users base their behavior on social media on trust in the medium. 
Therefore, we constructed a trust calculation model of adjacent users using three dimensions: 1) characteristic similarity trust $\operatorname{Ctr}(i, j), 2)$ interaction trust $\operatorname{Itr}(i, j)$, and 3) platform evaluation trust $\operatorname{Ptr}(i, j)$. The formula is

$$
\operatorname{Trust}(i, j)=\alpha \times \operatorname{Ctr}(i, j)+\beta \times \operatorname{Itr}(i, j)+\gamma \times \operatorname{Ptr}(i, j)
$$

Characteristic similarity trust is an indicator of a user's characteristics and refers to the user's trust in users with similar characteristics. We selected two indicators of characteristic similarity trust: gender and user QQ level. Gender was coded 0 (female) or 1 (male). Euclidean distance was used to calculate the Euclidean similarity function to measure the similarity of adjacent users. The value range was $[0,1]$.

$$
\operatorname{Ctr}(i, j)=\text { SimDistance }=\frac{1}{1+d_{a b}}, d_{a b}=\sqrt{\sum_{k=1}^{n}\left(x_{1 k}-x_{2 k}\right)^{2}}
$$

Interaction trust reflects the strength of the social relationship of adjacent users, represented as closeness $[29,30]$. It is the trust that users develop through interaction. Three factors (comments, @s to QQ friends, and messages) were used to calculate interaction trust between users [31]. The word comm indicates the comment ratio-the ratio of the number of comments that user $\mathrm{A}$ gives to user $\mathrm{B}$ to the total number of comments that B has received from B's QQ friends. The dummy variable mark refers to whether user B @s to QQ friends of user A in all the communications to user B. If user B @s to QQ friends of user $\mathrm{A}$, the value was 1 ; otherwise, the value was 0 . The term mes indicates the message ratio, which is the ratio of the number of messages sent by user $A$ to user B's message board to the total number of messages on B's message board in Qzone. The variables $\mu_{1}, \mu_{2}$, and $\mu_{3}$ indicate the weight of the three indicators. Because the three indicators together reflect the closeness of the relationship and have the same status, the definition of their weight is identical:

$\operatorname{Itr}(i, j)=\mu_{1} \times$ comm $+\mu_{2} \times$ mark $+\mu_{3} \times$ mes, $\mu_{1}=\mu_{2}=\mu_{3}=\frac{1}{3}$

User trust in social media is the basis for user trust in their friends. Thus, platform evaluation trust indicates the user's perception and trust in the Qzone platform [32]. Platform evaluation trust $\operatorname{Ptr}(i, j)$ is composed of three dimensions, namely: privacy protection evaluation, privacy [33,34], information credibility evaluation, information [35] , and user perceptual utility evaluation, utility [36,37], with a range of [0,
1]. Platform evaluation trust data were collected when the participants were recruited. The values of $\theta_{1}, \theta_{2}$, and $\theta_{3}$ indicate that the three dimensions are equally weighted:

$\operatorname{Ptr}(i, j)=\theta_{1} \times$ privacy $+\theta_{2} \times$ information $+\theta_{3} \times$ utility, $\theta_{1}=\theta_{2}=\theta_{3}=\frac{1}{3}$

$\alpha, \beta$, and $\gamma$ are the weights associated with three dimensions of trust (i.e., privacy, information, and utility, respectively) calculated using the structural entropy weight method. We first normalized the indicator, $x_{i j}^{\prime}=\frac{x_{i j}-\min \left(x_{i j}\right)}{\max \left(x_{i j}\right)-\min \left(x_{i j}\right)}$, then calculated the entropy and weight of the indicator. The user i's $\mathbf{j}$-th indicator weight is $x_{i j}^{\prime}$, and the information $y_{i j}=\frac{x_{i j}^{\prime}}{\sum_{i=1}^{m} x_{i j}^{\prime}}$

entropy of the j-th indicator is $e_{j}=-k \sum_{i=1}^{m} y_{i j} \ln y_{i j}, k=\frac{1}{\ln m}$

( $m$ is the number of indicators; $m=3$ ). We calculated the weight of the $\mathrm{j}$-th indicator, ${ }_{w_{j}}=\frac{1-e_{j}}{m-\sum e_{j}}$, obtaining the results of $\alpha=0.33, \quad \beta=0.32, \quad \gamma=0.35$.

\subsection{Setting the variables}

The indictor Trust was obtained using the trust calculation model. We then adopted an empirical approach to determine the factors that influence trust in social networks and the mechanisms of trust formation. Three key indicators, namely comm, mark, and mes, were selected. With this method, "B" is the sample user, and " $\mathrm{A}$ " is a QQ friend of user $\mathrm{B}$ - $\mathrm{a}$ user adjacent to $B$ :

$$
\begin{aligned}
& \text { comm }=\frac{\text { comamt }_{A B}}{\text { comamt }_{B}} \\
& \text { mes }=\frac{\text { mesamt }_{A B}}{\text { mesamt }_{B}}
\end{aligned}
$$$$
\operatorname{mark}= \begin{cases}0, & \text { have @ interaction } \\ 1, & \text { no @ interaction }\end{cases}
$$

We also selected control variables: comment refers to the number of comments from user A to user B, message refers to the number of messages that user $\mathrm{A}$ sends to user $\mathrm{B}$, comamt $_{B}$ refers to the total number of comments made by user $\mathrm{B}$, mesamt $_{B}$ refers to the total number of messages on user B's message board, 
degree $_{B}$ refers to the QQ level of user $\mathrm{B}$, and degree $_{A}$ refers to the QQ level of user A. The variables gender $_{B}$ and gender $_{A}$ are both dummy variables, representing the genders of user $\mathrm{A}$ and user $\mathrm{B}$ ( 0 for females and 1 for males). Table 2 provides the descriptive statistics of all the variables.

\section{Model construction and data analysis}

\subsection{Relationship between trust and closeness}

Through calculation, we obtained the indictor Trust. The control variables and factors influencing trust were taken from Qzone data of user B crawled by the Python program.

To understand how trust is formed between adjacent users in online social networks and to investigate the formation of trust in a virtual environment, we used the ordinary least squares (OLS) regression model to conduct the analysis. Trust, closeness, and other control variables (i.e., comm, mark, and mes) were the focus of attention. Table 3 presents the correlations between the three variables. Except for the dummy variable, the three key indicators are related to each other. Therefore, we included the three individual closeness indicators (comment ratio, message ratio, and a dummy variable, mark) in Model 1, Model 2, and Model 3, respectively, and then combined them in Model 4. Although we determined the correlation coefficients of the variables are significant, they cannot be substituted for each other. We used STATA 15.1 (StataCorp LLC, Texas, USA) for the OLS regression with trust, closeness, and control variables. The results are presented in Table 4. These results did not account for the problem of endogenous variables.

As Table 4 indicates, the relationships between comm, mark, and mes with trust are significant, as was expected. As the number of comments and messages by user A to his or her adjacent user B increases, B's trust in A increases, and an increase in @s to QQ friends by the two individuals also enhances their trust in each other. In multicollinearity tests, the variance expansion factor for each of the four models (see Table 4 for 4 models) was lower than the threshold of 10 , and thus, multicollinearity with the OLS regression was not a concern.

\subsection{Endogenous switching regression}

The sample size for the empirical study was small, and thus, it may not have been representative of the population. Random sampling would avoid selection bias $[38,39]$, but time and budget constraints prevented its use. Thus, we used convenience sampling. The self- selection may have estimation bias. This will be discussed later.

Endogeneity may be a problem with QQ users. Tencent QQ and Weibo are different social media. Weibo is a social medium for nonacquaintances, whereas QQ is used by acquaintances. In the case of $\mathrm{QQ}$, the majority of users know each other in real life. The estimation of trust over social media may not be precise because QQ users know each other in offline settings. An unobserved individual heterogeneity of user B may be related to an unobserved individual heterogeneity of user A. Thus, if users A and B are QQ friends, they may be quite similar. Consequently, the measurement of closeness may have a relatively high level of endogeneity. To better understand how trust between users is formed and developed, we introduced endogenous switching regression (ESR) to avoid estimation bias caused by self-selection and endogeneity.

Heterogeneity of user type may be another obstacle. We explored heterogeneity to further understand QQ users' trust formation. We analyzed the number of posts. We used a Python program to crawl the Qzone user data and analyzed these data. Users were divided into two categories based on the median of the level of interaction. One category comprises users whose dynamic quantity is larger than the average (users with a high level of interactions), and the other type consists of users whose level of interactions is lower than the average (user with a low level of interactions). The maximum likelihood estimation method based on a copula function was used to construct the ESR model [40]. For social network users, $S_{\mathrm{i}}$ is defined as the utility of the user's participation in social activities; it is an exogenous variable determined by the user's social behavior, and its value cannot be accurately observed. $Z$ is an n-dimensional vector representing the characteristics of social users, $\gamma$ is an $(n \times 1)$ dimensional column vector, $\mu$ is a random error term, Trust $_{M}$ is the trust of users with a larger dynamics quantity, Trust $_{F}$ is users' trust with a low level of interactions, and $S_{i}^{*}$ is the latent variable of the dummy variable $S_{\mathrm{i}}$. The discriminant function for classifying users according to the number of interactions sent by the user is

$$
S_{\mathrm{i}}^{*}=\delta\left(\text { Trust }_{M}-\text { Trust }_{F}\right)+Z_{i} \gamma+u_{i}
$$

The equation is defined according to the value of the latent variable $S_{\mathrm{i}}^{*}$ : 


$$
S_{i}=\left\{\begin{array}{l}
1, S_{i}^{*}>0 \\
0, S_{i}^{*} \leq 0
\end{array}\right.
$$

The symbol of the function enables us to understand the social behavior of the QQ. The equations constructed for users' trust on the social behavior of users are

Regime1: Trust $_{M}=\alpha+\beta_{1}$ comm $+\beta_{2}$ mes $+\beta_{3}$ mark $+\varepsilon_{1 i}, S_{i}=1$

Regime $2:$ Trust $_{F}=\alpha+\varphi_{1}$ comm $+\varphi_{2}$ mes $+\varphi_{3}$ mark $+\varepsilon_{2 i}, S_{\mathrm{i}}=0$

The model consists of a selection equation and two other equations, and the results of an estimate for a user can only appear in one result equation, not in both.

The model assumes error terms: $\mu_{i}, \varepsilon_{1 i}$, and ${ }^{\varepsilon_{2 i}}$, obeys a ternary normal distribution, and has a mean of zero.

The terms $\mu_{i}$, $\varepsilon_{1 i}$, and $\varepsilon_{2 i}$ are not independent of each other, as can be seen in the covariance matrix of the error term:

$$
\Omega=\left[\begin{array}{ccc}
\sigma_{u}^{2} & \sigma_{1 u} & \sigma_{2 u} \\
\sigma_{1 u} & \sigma_{1}^{2} & \cdot \\
\sigma_{2 u} & \cdot & \sigma_{2}^{2}
\end{array}\right]
$$

The independent variables in the model include three closeness indicators: comment ratio, message ratio, and a dummy variable, mark. These three variables are included in the ESR, and the interaction and pattern between trust and closeness in social networks can be found from differences in user type. The results of the ESR performed with STATA 15.1 for users with more interaction and with less interaction are presented in Table 5.

Model 5 is a switching regression for a user with fewer interactions. The sample size is 207 . The results suggest that user trust is significantly correlated with the dummy variable mark, and the effect of the comment ratio and the message ratio on trust is not significant. Thus, this type of user has fewer interactions and frequency of interaction with friends in Qzone is less than that of users who have more interactions. The interaction of comments and messages has a weak influence on the social connection between users and friends. Comments and messages show the activeness of a user's friends, and the user receives this information passively. However, the number of @s to QQ friends reveals a strong influence on a user's trust in his or her friends. We found that @s to QQ friends indicated a high degree of interaction, especially for those who do not have a lot of posts. The more @s to their friends the more trust to their friend. It is also likely that they encourage high level of offline contact with their QQ friends, not limited only having online activities with them. In Qzone interactions, a user who has more @s from QQ friends, he/she is highly trusted by his/her friends.

Model 6 generated the results of a switching regression for users who have more interactions with others. The sample size was 215 , and the results differed from those concerning users with fewer interactions. The trust of users with more interactions was positively correlated with the three types of closeness indicators. For such users, the level of interactions in Qzone was relatively high, and the frequency of interactions with friends was also relatively high. Such users tend to be more active in social networks, with more comment, message, and @s to QQ friends. These interactions build trust. Thus, the higher the number of social interactions with friends is, the more a user trusts those friends.

\subsection{Robustness test}

It is controversial whether an ESR model can completely solve the problem of endogeneity. We verified the solution to the problem through a robustness analysis. We selected "mesamt"; that is, "the number of messages user A gives to its adjacent user B," as the tool variable of the variable mes and employed two-stage least squares (2SLS) to test robustness. The results, shown in Table 6, suggest that the variable message has a positive effect on shaping trust between the two types of users, which further validates our results. According to the weak tool variable test, the value of the $F$ statistic is higher than 10 , and thus, selecting "mesamt" as a weak variable does not present a problem.

\section{Conclusion}

We constructed a model to assess the trust of social media users. Based on the data crawled by a Python program and the data generated from the model, we investigated factors that influence trust between adjacent users in social networks. First, OLS regression was performed to determine the relationship between trust and closeness in the Qzone social platform, and a linear relationship between trust and closeness was identified. The results of the OLS regression revealed that the trust of users in social networks is positively correlated with the three closeness indicators: comments, @s to QQ friends (a reminder nudge for attention), and messages. To solve the problem of endogeneity, an ESR was conducted to explore differences in trust formation with heterogeneous users. The users were classified based on level of interaction. The results of the ESR 
disclosed that the trust of users with a high level of interaction is positively related to the three closeness indicators, and the positive relationship between trust of users with a low level of interaction and the dummy variable mark is significant. Trust in social networks is closely related to the interaction of users. Finally, a robustness analysis using the 2SLS method with "mesamt" (the number of messages that user A gives to the adjacent user B) as a tool variable verified the results.

Trust in a social network has a positive relationship with the three closeness indicators proposed in this research. The improvement of closeness can promote trust in the social network. When using social media, users can improve their mutual trust by improving their closeness with friends. In addition, users who have more interactions and those who have fewer interactions are different in how they improve trust. Users with fewer interactions can focus on improving trust with friends by building relationships offline.

We combined mathematic models and empirical results to study trust on a social network. This research is subject to the following limitations. First, the sample size was small, and thus the problem of contingency cannot be ruled out. Large samples can increase statistical validity. Second, the sample may have had selection bias. Our participants were all college students. Factors affecting trust among social network users may differ in other age groups.

\section{References}

[1] China Internet Network Information Center (CNNIC). The 43rd Statistical Report on the Status of Internet Development in China. Retrieved on February 28, 2019.

[2] Baier, A. Moral prejudices: Essays on ethics. 1995, Harvard University Press.

[3] Antoci, A., Bonelli, L., Paglieri, F., Reggiani, T., and Sabatini, F. Civility and trust in social media. Journal of Economic Behavior \& Organization, 160, 2019, 83-99.

[4] Sako, M. Does trust improve business performance. Organizational trust: A reader, 2006, 88-117.

[5] Strauss, N. The role of trust in investor relations: a conceptual framework. Corporate Communications: An International Journal, 23(1), 2018, 2-16.

[6] Yadav, A., Chakraverty, S., and Sibal, R. A framework for classifying trust for online systems. World Wide Web, 22(4), 2019, 1499-1521.

[7] Schurr, P. H. and Ozanne J. L. Influences on exchange processes: Buyers' preconceptions of a seller's trustworthiness and bargaining toughness. Journal of Consumer Research, 1985, 11(4).

[8] Anderhub, V., Müller, R., and Schmidt, C. Design and evaluation of an economic experiment via the Internet. Journal of Economic Behavior \& Organization, 46(2), 2001, 227-247.

[9] Riyanto, Y. E., and Jonathan, Y. X. Directed trust and trustworthiness in a social network: An experimental investigation. Journal of Economic Behavior \& Organization, 151, 2018, 234-253.

[10] Urena, R., Kou, G., Dong, Y., Chiclana, F., and Herrera-Viedma, E. A review on trust propagation and opinion dynamics in social networks and group decision making frameworks. Information Sciences, 478, 2019, 461-475.

[11] Sabatini, F. and Sarracino, F. Online social networks and trust. Social Indicators Research, 142(1), 2019, 229-260.

[12] Akilal, K., Slimani, H., and Omar, M. A very fast and robust trust inference algorithm in weighted signed social networks using controversy, eclecticism, and reciprocity. Computers \& Security, 83, 68-78.

[13] Golzardi, E., Sheikhahmadi, A., and Abdollahpouri, A. Detection of trust links on social networks using dynamic features. Physica A: Statistical Mechanics and its Applications, 527, 2019, 121-269.

[14] Laifa, M., Akrouf, S., and Mammeri, R. Forgiveness and trust dynamics on social networks. Adaptive Behavior, 26(2), 2018, 65-83.

[15] Frey, V., Buskens, V., and Corten, R. Investments in and returns on network embeddedness: An experiment with trust games. Social Networks, 56, 2019, 81-92.

[16] Xu, L., Bao, T., Zhu, L., and Zhang, Y. (). TrustBased Privacy-Preserving Photo Sharing in Online Social Networks. IEEE Transactions on Multimedia, 21(3), 2018, 591-602.

[17] Philippe, R. A. and Seiler, R. Closeness, coorientation and complementarity in coach - athlete relationships: What male swimmers say about their male coaches. Psychology of Sport \& Exercise, 7(2), 2006, 159-171.

[18] Asim, Y., Malik, A. K., Raza, B., and Shahid, A. R. A trust model for analysis of trust, influence and their relationship in social network communities. Telematics and Informatics, 36, 2019, 94-116.

[19] Ali-Eldin, A. M. Trust prediction in online social rating networks. Ain Shams Engineering Journal, 9(4), 2018, 3103-3112.

[20] Ntwiga, D. B., Weke, P., and Kirumbu, M. K. Trust model for social network using singular value decomposition. Interdisciplinary Description of Complex Systems: INDECS, 14(3), 2016, 296-302.

[21] Bapna, R., Gupta, A., Rice, S., and Sundararajan, A. Trust and the Strength of Ties in Online Social Networks: An Exploratory Field Experiment. MIS 
Quarterly, 41(1), 2017, 115-130.

[22] Park, S., Park, M., Kim, H., Kim, H., Yoon, W., Yoon, T. B., and Kim, K. P. A closeness centrality analysis algorithm for workflow-supported social networks. In 2013 15th International Conference on Advanced Communications Technology (ICACT), 2013, January, 158-161.

[23] Khopkar, S. S., Nagi, R., Nikolaev, A. G., and Bhembre, V. Efficient algorithms for incremental all pairs shortest paths, closeness and betweenness in social network analysis. Social Network Analysis and Mining, 4(1), 2014, No. 220.

[24] Shen, C. and Gong, H. Personal ties, group ties and latent ties: connecting network size to diversity and trust in the mobile social network WeChat. Asian Journal of Communication, 29(1), 2019, 18-34.

[25] Lin, Y., Zhang, J., Ying, Y., Hong, S., and Li, H. FVBM: A Filter-Verification-Based Method for Finding Top-k Closeness Centrality on Dynamic Social Networks. In Asia-Pacific Web Conference, 2016, September , 389-392, Springer, Cham.

[26] Zhang, J., and Luo, Y. Degree Centrality, Betweenness Centrality, and Closeness Centrality in Social Network. In 2017 2nd International Conference on Modelling, Simulation and Applied Mathematics (MSAM2017), 2017, March, Atlantis Press.

[27] Moore, J., Carrasco, J. A., and Tudela, A. Exploring the links between personal networks, time use, and the spatial distribution of social contacts. Transportation, 40(4), 2013, 773-788.

[28] Zhan, J. and Fang, X. A novel trust computing system for social networks. In 2011 IEEE Third International Conference on Privacy, Security, Risk and Trust and 2011 IEEE Third International Conference on Social Computing, 2011, October, 1284-1289, IEEE.

[29] Kang, K., Kwak, M., and Shin, S. K. A Calculus of Virtual Community Knowledge Intentions: Anonymity and Perceived Network-Structure. Journal of Computer Information Systems, 58(2), 2018, 110121.

[30] Parks, M. R. and Floyd, K. Meanings for closeness and intimacy in friendship. Journal of Social \& Personal Relationships, 13(1), 1996, 85-107.

[31] Koshutanski, H., and Massacci, F. An interactive trust management and negotiation scheme. In IFIP World Computer Congress, TC 1, 2004, August, 115128, Springer, Boston, MA.

[32] Ginsberg, M. U.S. Patent No. 7,284,124. Washington, DC: U.S., 2007, Patent and Trademark Office.

[33] Fogel, J., and Nehmad, E. Internet social network communities: Risk taking, trust, and privacy concerns. Computers in Human Behavior, 25(1), 2009, 153-160.

[34] Young, A. L. and Quan-Haase, A. Information revelation and internet privacy concerns on social network sites: a case study of facebook. In Proceedings of the fourth international conference on Communities and technologies, 2009 June, 265-274. ACM.

[35] Castillo, C., Mendoza, M., and Poblete, B. Information credibility on twitter. In Proceedings of the 20th international conference on World Wide Web, 2011, March, 675-684, ACM.

[36] Hsu, C. L., and Lin, J. C. C. Exploring factors affecting the adoption of Internet of Things services. Journal of Computer Information Systems, 58(1), 2018, 49-57.

[37] Beach, S. R., and Arias, I. Assessment of perceptual discrepancy: Utility of the primary communication inventory. Family process, 22(3), 1983, 309-316.

[38] Cooper, R. On allocative distortions in problems of self-selection. The Rand Journal of Economics, 15(4), 1984, 568-577.

[39] Shanmugam, K. R. Self selection bias in the estimates of compensating differentials for job risks in India. Journal of Risk and Uncertainty, 22(3), 2001, 263-275.

[40] Hasebe, T. Copula-based maximum-likelihood estimation of sample-selection models. The Stata Journal, 13(3), 2013, 547-573.

[41] Sina Finance. Tencent Financial Report, (2019, March, 19th), Retrieved from,

http://finance.sina.com.cn/stock/stockzmt/2020-0319/doc-iimxyqwa1525624.shtml

[42] CNNIC (2020, April, 28 $\left.{ }^{\text {th }}\right)$. The $45^{\text {th }}$ China Statisicial Report on Internet Development, Retrieved from, http://www.cac.gov.cn/202004/27/c_1589535470378587.htm 


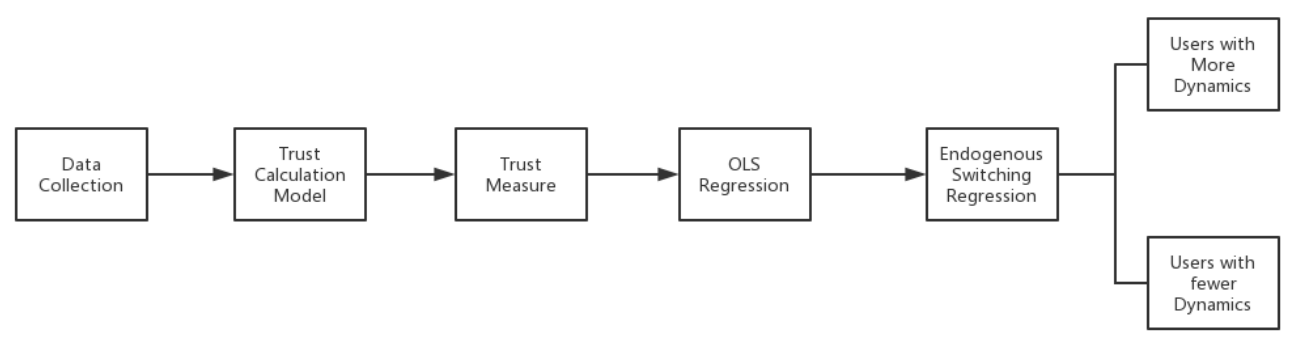

Figure 1. Research framework

Table 1 Demographics of Participants

\begin{tabular}{|c|c|c|c|}
\hline \multirow{2}{*}{ Year in College } & Category & Number & Percentage \\
\cline { 2 - 4 } & & 108 & $72 \%$ \\
\cline { 2 - 4 } & Senior & 24 & $16 \%$ \\
\hline \multirow{2}{*}{ Gender } & Granior & 18 & $12 \%$ \\
\cline { 2 - 4 } & Male & 84 & $56 \%$ \\
\hline \multirow{2}{*}{ Age } & Female & 66 & $44 \%$ \\
\cline { 2 - 4 } & over 22 years old & 132 & $88 \%$ \\
\hline \multirow{2}{*}{ Experience } & $3-5$ years & 18 & $12 \%$ \\
\cline { 2 - 4 } & 5-10 years & 25 & $17 \%$ \\
\cline { 2 - 4 } & Over 10 years & 89 & $59 \%$ \\
\hline
\end{tabular}

Table 2. Descriptive Statistics

\begin{tabular}{|l|r|r|r|r|r|}
\hline Variable & Count & \multicolumn{1}{l|}{ Mean } & \multicolumn{1}{l|}{ Std. Dev. } & Min & Max \\
\hline Trust & 422 & 0.3286 & 0.1165 & 0.4889 & 0.7946 \\
\hline comm & 422 & 0.0398 & 0.0479 & 0.0034 & 0.4157 \\
\hline mark & 422 & 0.4561 & 0.4987 & 0 & 1 \\
\hline mes & 422 & 0.0363 & 0.0470 & 0 & 0.4286 \\
\hline comment & 422 & 4.3460 & 4.5754 & 1 & 37 \\
\hline comamt $_{B}$ & 422 & 134.4242 & 66.7731 & 15 & 293 \\
\hline message $^{\text {mesamt }}$ & 422 & 4.9929 & 6.7604 & 0 & 59 \\
\hline degree $_{B}$ & 422 & 163.8365 & 136.7147 & 18 & 630 \\
\hline degree $_{A}$ & 422 & 57.0380 & 9.6538 & 32 & 82 \\
\hline gender $_{B}$ & 422 & 55.9123 & 6.5937 & 36 & 73 \\
\hline gender $_{A}$ & 422 & 0.4479 & 0.4979 & 0 & 1 \\
\hline
\end{tabular}

Table 3. Correlation Coefficients of Independent Variables

\begin{tabular}{|l|l|l|l|}
\hline Variable & comm & mes & mark \\
\hline comm & 1.000 & & \\
\hline
\end{tabular}




\begin{tabular}{|l|l|l|l|}
\hline mes & $0.211^{* * *}$ & 1.000 & \\
\hline mark & $0.147 * * *$ & 0.076 & 1.000 \\
\hline
\end{tabular}

Note: ${ }^{*} \mathrm{p}<0.05, * * \mathrm{p}<0.01, * * * \mathrm{p}<0.001$

Table 4 Relationship between Trust and Closeness

\begin{tabular}{|c|c|c|c|c|c|c|c|c|}
\hline & \multicolumn{2}{|c|}{ Model 1} & \multicolumn{2}{|c|}{ Model 2} & \multicolumn{2}{|c|}{ Model 3} & \multicolumn{2}{|c|}{ Model 4} \\
\hline & Coeff & $\mathrm{p}>\mathrm{t}$ & Coeff & $\mathrm{p}>\mathrm{t}$ & Coeff & $p>t$ & Coeff & $\mathrm{p}>\mathrm{t}$ \\
\hline comm & 0.7544 & $0.000 * * *$ & & & & & 0.4837 & $0.000 * * *$ \\
\hline mark & & & 0.1245 & $0.000 * * *$ & & & 0.1161 & $0.000 * * *$ \\
\hline mes & & & & & 0.4695 & $0.000 * * *$ & 0.2676 & $0.009 * * *$ \\
\hline comamt & 0.0001 & 0.875 & -0.0001 & 0.140 & -0.0002 & 0.096* & -0.0001 & 0.791 \\
\hline mesamt & 0.0001 & $0.008 * * *$ & 0.0001 & $0.029 * *$ & 0.0001 & $0.003 * * *$ & 0.0001 & $0.005 * * *$ \\
\hline degree $_{B}$ & -0.0011 & $0.09 * *$ & -0.0001 & $0.067 *$ & -0.0012 & 0.058* & -0.0012 & $0.021 * *$ \\
\hline degree $_{A}$ & 0.0012 & 0.145 & 0.0011 & 0.164 & 0.0011 & 0.225 & 0.0012 & 0.111 \\
\hline gender $_{B}$ & 0.0022 & 0.856 & 0.0030 & 0.778 & 0.0033 & 0.796 & -0.0022 & 0.835 \\
\hline gender $_{A}$ & 0.0043 & 0.689 & 0.0011 & 0.912 & 0.0092 & 0.411 & 0.0008 & 0.928 \\
\hline constant & 0.2629 & $0.000 * * *$ & 0.2694 & $0.000 * * *$ & 0.3121 & $0.000 * * *$ & 4.675 & $0.000 * * *$ \\
\hline $\mathrm{R}^{2}$ & & 0.0862 & & 0.2978 & & 0.0461 & & 0.2361 \\
\hline VIF & & 1.22 & & 1.18 & & 1.19 & & 1.20 \\
\hline
\end{tabular}

Note: ${ }^{*} \mathrm{p}<0.05, * * \mathrm{p}<0.01, * * * \mathrm{p}<0.001$

Table 5. The Results of Endogenous Switching Regression

\begin{tabular}{|c|c|c|c|c|}
\hline Sample & \multicolumn{2}{|c|}{ Model 5(users with less interactions ) } & \multicolumn{2}{|c|}{ Model 6(users with more interactions) } \\
\hline $\begin{array}{l}\text { dependent } \\
\text { variable }\end{array}$ & \multicolumn{2}{|c|}{ Trust } & \multicolumn{2}{|c|}{ Trust } \\
\hline $\begin{array}{l}\text { independent } \\
\text { variable }\end{array}$ & coefficient & $\mathrm{p}>|\mathrm{z}|$ & coefficient & $\mathrm{p}>|\mathrm{z}|$ \\
\hline comm & 0.3978 & 0.160 & 0.4199 & $0.030 * *$ \\
\hline mes & 0.1923 & 0.212 & 0.2252 & $0.087 *$ \\
\hline mark & 0.1253 & $0.001^{* * *}$ & 0.1169 & $0.000^{* * *}$ \\
\hline constant & 0.2715 & $0.000 * * *$ & 0.2414 & $0.002 * * *$ \\
\hline Counts & \multicolumn{2}{|c|}{207} & \multicolumn{2}{|c|}{215} \\
\hline
\end{tabular}

Note: ${ }^{*} \mathrm{p}<0.05, * * \mathrm{p}<0.01, * * * \mathrm{p}<0.001$

Table 6. Results of 2SLS Regression

\begin{tabular}{|c|c|c|c|}
\hline Model & $\begin{array}{c}\text { Users with more } \\
\text { interactions } \\
\text { (Model 7) }\end{array}$ & $\begin{array}{c}\text { Users with less interactions } \\
\text { (Model 8) }\end{array}$ & $\begin{array}{c}\text { Overall } \\
\text { (Model 6) }\end{array}$ \\
\hline comm & $0.9690^{* * *}$ & $0.5153^{* * *}$ & -3.58534 \\
\hline mes & $-2.2984^{*}$ & -0.7884 & $0.1473^{* * *}$ \\
\hline constant & $0.1297^{* * *}$ & $0.1192^{* * *}$ & $0.3507^{* * *}$ \\
\hline
\end{tabular}

Note: ${ }^{*} \mathrm{p}<0.05, * * \mathrm{p}<0.01, * * * \mathrm{p}<0.001$ 